\title{
Roughening transition, surface tension and equilibrium droplet shapes in a two-dimensional Ising system $\dagger$
}

\author{
J E Avron $\ddagger$, H van Beijeren§, L S Schulman\| and R K P Zia \\ ¥ Department of Physics, California Institute of Technology Pasadena, CA 91125, USA \\ \$Institut für Theoretische Physik, Rheinisch Westfälische Technische Hochschule, \\ Aachen, FRG \\ || IBM Research Center, Yorktown Heights, NY 10598, USA, and Physics Department, \\ Technion, Haifa, Israel \\ II Institüt für Festkörperforschung, KFA -Jülich, D-5170, FRG, and Physics Department, \\ Virginia Polytechnic Institute and State University, Blacksburg, VA 24061, USA
}

Received 27 October 1981

\begin{abstract}
The exact surface tension for all angles and temperatures is given for the two-dimensional square Ising system with anisotropic nearest-neighbour interactions. Using this in the Wulff construction, droplet shapes are computed and illustrated. Letting temperature approach zero allows explicit study of the roughening transition in this model. We also compare our results with those of the solid-on-solid approximation.
\end{abstract}

\section{Introduction}

Recently the roughening transition associated with the disappearance of a sharply defined orientation of an interface has received increasing interest. It has been recognised that this phase transition may influence crystal morphology as well as the process of crystal growth (Leamy et al 1975). Furthermore, it has been discovered that the roughening transition has close connections to phase transitions in other twodimensional models, e.g. the $X Y$ model (José et al 1977, Knops 1977), the Coulomb gas (Chui and Weeks 1976) and the sine-Gordon model (Müller-Krumbhaar 1977), as well as in lattice gauge theories.

Experimentally, the roughening transition is not yet well established. It is apparently difficult to find interfaces having a roughening transition within an admissible parameter range. Moreover, for most crystals, surface equilibrium phenomena are greatly affected by tiny deviations from equilibrium in the bulk. The first experiments in which a sudden transition from flat to rounded interfaces at a fairly sharply defined temperature was seen, were performed by Pavlovska and Nenov (1971a, b, 1977) on negative crystals in naphthalene and diphenyl. Recently similar observations on interfaces between solid and superfluid helium, a system for which extremely good conditions of purity and equilibration can be obtained, were reported by Avron et al (1980). 
In computer experiments on solid-on-solid models the existence of a roughening transition has been shown fairly convincingly; moreover, for certain of these models the existence of a roughening transition could be shown rigorously (van Beijeren 1977) or at least beyond reasonable doubt (Chui and Weeks 1976). The problem with the real experiments, however, is ambiguity in the interpretation of the observed phenomena as a roughening transition. One question is whether one should expect to see a sudden change of crystal shape at a roughening transition or instead a gradual transition from a perfectly faceted shape to a slightly rounded form. Moreover, the roughening transition is expected to be markedly affected by gravity and as yet there is no theory which incorporates gravity. It may turn out that the above two difficulties are, in fact, related.

To address this question we consider the equilibrium shape of a 'droplet' in the two-dimensional Ising model with ferromagnetic nearest-neighbour interactions. For the isotropic case (equal coupling constants in both directions) Abraham and Reed (1977) calculated the interface free energy per unit length (the surface tension) as a function of the direction of the interface. From this, the shape of the interface can be obtained directly by means of the Wulf construction. In this note we extend this result to the general anisotropic case and formulate it in more explicit form. We also provide computer plots of the equilibrium shape which show that at low temperatures it is nearly square or rectangular (the equilibrium shape at $T=0$ ) even though the interface is 'rough' for all $T>0$. For low temperatures we make a comparison between the exact results and those of the solid-on-solid approximation obtained by Burton et al (1951). The latter results, which can be obtained very simply, turn out to be accurate to order $\exp (-$ const $/ T)$.

In passing, we note the equivalence for the two-dimensional Ising model of the common definitions of the roughening transition, namely (i) the temperature above which the interface is not localised so that its 'width' $\Delta$ diverges in the thermodynamic limit, and (ii) the temperature above which the free energy of a 'step' in the interface vanishes in the thermodynamic limitt. Intuitively the equivalence of these two definitions is rather clear: If the formation of a step in the interface requires no free energy, the interface will in general contain a large number of steps, which will delocalise its precise position in the orthogonal direction. Furthermore, the vanishing of the step free energy implies that the discontinuity in the derivative of the interface free energy with respect to its direction vanishes for the direction under consideration. This means that the Wulfi plot has no 'kink' for this direction, hence the interface has no facets orthogonal to it. Another point of some importance is that although the interfacial width $\Delta$ 'diverges' for $T>T_{R}$, it is meaningful to speak of an equilibrium shape of a droplet. It is known that $\Delta$ diverges with $L$, the size of the system. Within capillary wave theories

$$
\Delta / L \propto L^{1 d \cdot 11 / 2} \quad d<3
$$

for a $(d-1)$-dimensional interface embedded in a $d$-dimensional bulk system. In two dimensions, explicit calculations of Abraham and Reed (1977) vindicate (1) and give the temperature-dependent constant of proportionality. Thus on a scale of $L^{1 / 2}$ the width has finite thermodynamic limit and for a droplet of size $L$ the shape is well defined. Therefore it is meaningful to speak of the average shape of a dropiet for all $T<T_{c}$, where $T_{c}$ is the critical temperature.

t The free energy of a step in an interface, orthogonal say to the $y$ direction, can be defined as the difference in free energy between an interface for which the $y$ coordinates of the end points differ by one lattice unit and an interface for which the end points have the same $y$ coordinates. 


\section{Equilibrium shape of a droplet}

The equilibrium shape of a 'droplet' of negative magnetisation in an environment of positive magnetisation is obtained by applying the Wulff construction (see Herring 1975). To be specific, let $\sigma(\hat{n})$ denote the free energy per unit length of an interface orthogonal to $\hat{n}$. Construct a line normal to $\hat{n}$ at a distance $\sigma(\hat{n})$ from the origin. Do this for all $\hat{n}$. Then the inner envelope of all lines drawn defines the equilibrium shape of the droplet.

For the two-dimensional Ising model with ferromagnetic nearest-neighbour couplings $J_{x}$ and $J_{y}$ the function $\sigma(\hat{n}) \equiv \sigma(\phi)$ (where $\phi$ is the angle between $\hat{n}$ and the $y$ axis) may be obtained from the duality relation (Fisher 1969, Watson 1972, Zia 1978)

$$
\beta \xi^{*}(\phi) \sigma(\phi)=1
$$

where $\xi^{*}$ is the correlation length in the direction orthogonal to $\hat{n}$ (i.e. along the interface) in the dual system. The latter may be extracted from the asymptotic behaviour of the correlation function of a spin at the origin $(0,0)$ of the lattice and another at $(M \cos \phi, M \sin \phi)$ :

$$
1 / \xi^{*}(\phi)=\lim _{M \rightarrow \infty}(-1 / M) \ln \langle S(0,0) S(M \cos \phi, M \sin \phi)\rangle .
$$

Asymptotic forms of $\langle S S\rangle$ have been computed exactly (Cheng and Wu 1967, see also McCoy and Wu 1973) so that explicitly,

$$
\beta \sigma(\phi)=\alpha_{1} \sin \phi+\alpha_{2} \cos \phi
$$

where

$$
\begin{aligned}
& p \cosh \alpha_{1}=\left[\left(a^{2}-q^{2}\right) \sin ^{2} \phi+p^{2} \cos ^{2} \phi\right] /\left[a \sin ^{2} \phi+b\right] \\
& q \cosh \alpha_{2}=\left[\left(a^{2}-p^{2}\right) \cos ^{2} \phi+q^{2} \sin ^{2} \phi\right] /\left[a \cos ^{2} \phi+b\right]
\end{aligned}
$$

and

$$
\begin{aligned}
& b \equiv\left\{\left(\frac{1}{2} a \sin 2 \phi\right)^{2}+\cos 2 \phi\left[p^{2} \cos ^{2} \phi-q^{2} \sin ^{2} \phi\right]\right\}^{1 / 2} \\
& a \equiv\left(1+x^{2}\right)\left(1+y^{2}\right) \quad p \equiv 2 x\left(1-y^{2}\right) \quad q \equiv 2 y\left(1-x^{2}\right) \\
& x \equiv \exp \left(-2 \beta J_{x}\right) \quad y \equiv \exp \left(-2 \beta J_{y}\right) .
\end{aligned}
$$

In the isotropic case $\left(J_{x}=J_{y}\right)$ these results agree with those of Abraham and Reed (1977).

From these equations we solved numerically for the equilibrium shape of the droplet, with the following results:

(a) At $T=0$ the Wulff plot $\sigma(\phi)$ consists of the outer envelope of four intersecting ellipses centred at $(x, y)=\left( \pm J_{x}, \pm J_{y}\right)$ and is given by

$$
\sigma(\phi)=2\left[J_{y}|\cos \phi|+J_{x}|\sin \phi|\right] \text {. }
$$

There are four kinks located at $\phi=0, \pi, \pm \frac{1}{2} \pi$ and the equilibrium shape is a rectangle. The anisotropic shape is obtained from the isotropic one by capillary wave scaling $x \rightarrow J_{x}^{-1} x, y \rightarrow J_{y}^{-1} y$.

(b) For $0<T<T_{\mathrm{c}}$ the Wulff plot $\sigma(\phi)$ has no kinks, so there are no facets on the equilibrium droplet. This establishes that $T_{R}^{\mathrm{II}}$, the roughening temperature defined according to the second definition given above, equals zero for all directions, and it confirms the equivalence of $T_{R}^{\mathrm{I}}$ and $T_{R}^{\mathrm{II}}$ for this case. (The result $T_{R}^{\mathrm{I}}=0$ is known from 
Gallavotti (1972), Abraham and Reed (1974, 1976) and Aizenmann (1980).) Furthermore, the equilibrium shape of the droplet is everywhere smooth (has no sharp angles) for $0<T<T_{\mathrm{c}}$. This is intuitively appealing; the interface is a one-dimensional structure with short-ranged interactions for which one would not expect any nonanalytic behaviour except at $T=0$. However, a general proof that no sharp angles can occur is not known to us.

(c) In figure 1 the equilibrium shape of the droplet is shown in the isotropic case $\left(J_{x}=J_{y}\right)$ for a number of temperatures. One sees that at a temperature of $0.1 T_{c}$ the equilibrium shape is already nearly square. This is in agreement with earlier results of Minlos and Sinai $(1967,1968)$, who proved that at very low temperatures the deviations from a square shape in the isotropic case are minor.

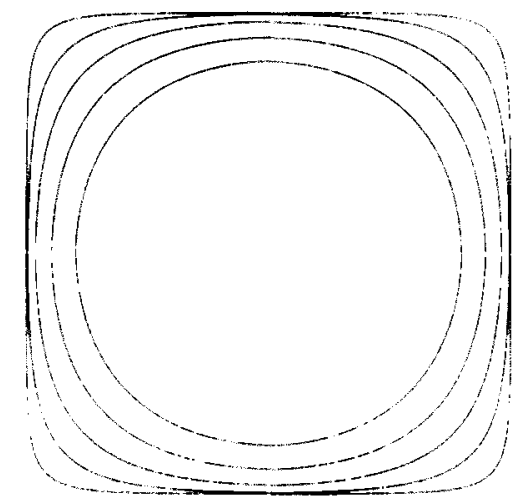

Fegure 1. Equilibrium shapes for isotropic couplings at $T / T_{\mathrm{c}}=0.1,0.2,0.3,0.4$ and 0.5 , with the larger figures being lower temperatures.

(d) As $T \rightarrow 0$ the curvature $\mathrm{d}^{2} \sigma / \mathrm{d} \phi^{2}$ diverges exponentially at the kink sites $\phi=0, \pi, \pm \frac{1}{2} \pi$, e.g., like $k T \exp \left(2 \beta J_{x}\right)$ for $\phi=\frac{1}{2} \pi$.

In figure 2, we provide plots of both $\sigma$ and the equilibrium shape in one quadrant for some typical temperatures. The behaviour of the equilibrium shape at general temperatures is also interesting; it turns out for instance that the anisotropic case can no longer be mapped onto the isotropic case by capillary wave scaling, or even, if one looks precisely, by any other scaling. This will be treated however in a separate paper (Zia and Avron, in preparation).

\section{The solid-on-solid (sos) approdimation}

This approximation treats the phase boundary as a contour between areas of positive and negative magnetisation that when followed from one end to the other is only allowed to move in three of the four possible directions. The interactions between this contour and the spins in the bulk are neglected, which implies that the probability for a given contour to occur is proportional to its Boltzmann weight exp $\left(-\beta E_{\mathrm{c}}\right)$ with $E_{\mathrm{c}}=2 J_{y} N_{\mathrm{h}}+2 J_{x} N_{\mathrm{v}} ; N_{\mathrm{h}}$ is the total length of horizontal line pioces measured in lattice units in the contour and $N_{v}$ the total length of vertical line pieces. The free energy per unit length, under the constraint that the average angle of the interface with the $y$ 


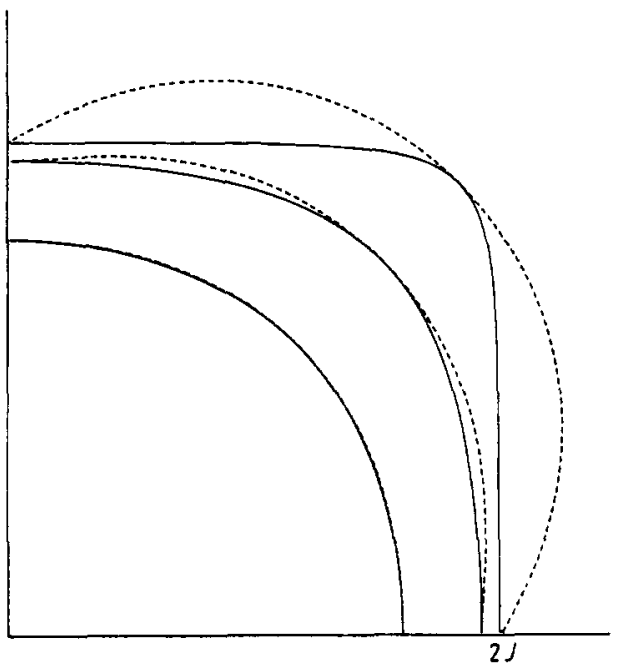

Figure 2. The full curve gives equilibrium shapes and the broken curve surface tension for $T / T_{\mathrm{c}}=0.1,0.3$ and 0.5

direction equals $\phi$, is found as (Burton et al 1951)

$\sigma^{\text {sos }}=f(\phi)=-k T|\cos \phi| \ln \left\{\exp \left(-2 \beta J_{y}\right) \sum_{n_{y}=-\infty}^{\infty} \exp \left(-2 \beta\left|n_{y}\right| J_{x}\right) \exp \left(h n_{y}\right)\right\}$

with a Lagrange multiplier $h$ to be determined from $\left\langle n_{y}\right\rangle=\tan \phi$. The explicit solution of this problem is straightforward. It was first given by Burton et al (1951) and reads

$$
\begin{aligned}
f(\phi)=2 J_{y}|\cos \phi|+2 J_{x}|\sin \phi|+k T\left[|\sin \phi| \log \left(\frac{|\sin \phi|}{|\sin \phi|+|\cos \phi|}\right)\right. \\
\left.\quad+|\cos \phi| \log \left(\frac{|\cos \phi|}{|\sin \phi|+|\cos \phi|}\right)\right]+k T\left\{|\cos \phi| \log \left(\frac{1+\varepsilon-R}{(1-\varepsilon)(1-|\tan \phi|)}\right)\right. \\
\left.+|\sin \phi| \log \frac{1}{2}(1+\varepsilon+R|\cot \phi|)\right\}
\end{aligned}
$$

where $\varepsilon=\exp \left(-4 \beta J_{x}\right), R=\left\{\tan ^{2} \phi(1-\varepsilon)^{2}+4 \varepsilon\right\}^{1 / 2}$ and we have chosen the parameters of this system to fit the couplings $J_{x}, J_{y}$ of the Ising model. The accuracy of the sos approximation can be checked by comparing $f(\phi)$ with $\sigma(\phi)$. Though tedious, the computations are straightforward. They lead to the well known result (Temperley 1952) $f(0)=\sigma(0)$ for all $T<T_{\text {c. }}$. For $\phi \neq 0, \sigma(\phi) \neq f(\phi)$; but for low temperatures the sos approximation turns out to be extremely good for $|\phi| \leqslant \frac{1}{4} \pi$. For larger angles it makes more sense to use the sos approximation in the orthogonal direction, although one introduces small spurious kinks in the Wulff plot by this procedure. The difference $f(\phi)-\sigma(\phi)$ is $\mathrm{O}\left(T \exp \left(-4 J_{y} / k T\right)\right)$ for small $T$, and hence approaches 0 very rapidly as $T$ tends to zero. In the isotropic case and for $\phi=\frac{1}{4} \pi, f$ approximates $\sigma$ within $1 \%$ up to $T=0.5 T_{\text {c. }}$. It is noteworthy in this context that the main two contributions to $f(\phi)$ can be recognised as zero-point-energy (the first two terms in (7)) and $(-T)$ times zeropoint-entropy (the third term in (7)). The remaining contributions are of order $T \varepsilon$ for 
$\phi \neq 0$, but are essential for cancelling the kink in the Wulff plot at $\phi=0$ that would result from the first three terms alone.

At higher temperatures, especially near $T_{\mathrm{c}}$, the sos approximation is in general no longer very good.

Further results will be published elsewhere (Zia and Avron, in preparation).

Finally, we want to mention that we received a preprint by $C$ Rottman and $M$ Wortis (1981) on the same subject after finishing this Letter.

We wish to acknowledge useful discussions with K Binder, M E Fisher and J Slawny. We are grateful to A Dekel for help with computers.

\section{References}

Abraham D B and Reed P 1974 Phys. Rev. Lett. 33377

1976 Commun. Math. Phys. 4935

1977 J. Phys. A: Math. Gen. 10 L121

Aizenmann M 1980 Commun. Math. Phys. 7383

Avron J E, Balfour L S, Kuper C G, Landau J, Lipson S G and Schulman L S 1980 Phys. Rev. Lett. 45814 van Beijeren H 1977 Phys. Rev. Lett. 38993

Burton W K, Cabrera N and Frank F C 1951 Phil. Trans. R. Soc. 243299

Cheng H and Wu T T 1967 Phys. Rev. 164719

Chui S T and Weeks J D 1976 Phys. Rev. B 144978

Fisher M 1969 J. Phys. Soc. Japan (suppl.) 2687

Gallavotti G 1972 Commun. Math. Phys. 27103

Herring C 1975 in Structure and Properties of Solid Surfaces ed R Gomer and G S Smith (Chicago: University of Chicago Press)

José J V, Kadanoff L P, Kirkpatrick S and Nelson D 1977 Phys. Rev. B 16127

Knops H J F 1977 Phys. Rev. Lett. 39766

Leamy H J, Gilmer G H and Jackson K A 1975 in Surface Physics of Materials vol 1, ed J M Blakely (New York: Academic)

McCoy B M and Wu T T 1973 The Two Dimensional Ising Model (Cambridge, Mass.: Harvard University Press)

Minlos R A and Sinai Ja G 1967 Math. USSR-Sbornik 2335 1968 Trans. Moscow Math. Soc. 19121

Müller-Krumbhaar H 1977 Developments in the theory of the roughening transition in Crystal Growth and Materials ed E Kaldis and H Y Scheel (Amsterdam: NHPC)

Pavlovska A and Nenov D 1971a Surface Sci. 27211

- 1971 b J. Crystal Growth 129 1977 J. Crystal Growth 39376

Rottman C and Wortis M 1981 Phys. Rev. B, in press

Temperley H N V 1952 Proc. Camb. Phil. Soc. 48683

Watson P G 1972 in Phase Transitions and Critical Phenomena vol 2, ed C Domb and M S Green (New York: Academic)

Zia R K P 1978 Phys. Lett. A 64345 